\title{
FREQUENCY-DEPENDENT POLARIZATION IN \\ COMPTONIZATION MODELS FOR AGN
}

\author{
JURI POUTANEN \\ Observatory and Astrophysics Laboratory, P.O.Box 14, SF-00014 University of Helsinki, Finland
}

\begin{abstract}
The angular distribution and the polarization of radiation as a function of the angle and frequency for the two-phase model of accretion disks in AGN are found. The results depend strongly on the temperature of the hot corona.
\end{abstract}

Key words: accretion disks, polarization, scattering

Two-phase accretion disk models have been used to explain the X-ray emission from galactic black hole candidates (Bisnovatyi-Kogan and Blinnikov, 1977) and AGNs (Czerny and Elvis, 1987; Wandel and Liang, 1991; Haardt and Maraschi, 1993). In such models soft radiation emitted by cool optically thick disk scatters in hot optically thin corona and gives rise to the power-law X-ray spectrum.

Taking into account only these two components I calculate the polarization degree in UV and X-ray region as a function of frequency and inclination angle of the disk. Such calculation in the single scattering approximation were performed by Poutanen and Vilhu (1993). Radiative transfer equation for Compton scattering of polarized light is formulated by Nagirner and Poutanen (1993a,b). For the case of the plane-parallel atmosphere this equation is solved by the iteration method (Sunyaev and Titarchuk, 1985).

The results show that polarization degree decreases when the electron temperature of the corona increases. The exact Compton scattering matrix predicts much smaller degree of linear polarization than the Rayleigh matrix does (Haardt and Matt, 1993). In the energy band where first scattering is important polarization depends on the angular and polarization properties of initial radiation from cool disk. The polarization angle in hard X-ray region is orthogonal to that in UV region. Future polarimetric observations in UV and X-ray regions need the theory of multiple Compton scattering of polarized light taking into account the exact scattering matrix.

\section{References}

Bisnovatyi-Kogan, G.S. and Blinnikov, S.I.: 1977, Astron.Astrophys. 59, 111

Czerny, B. and Elvis, M.: 1987, Astrophys.J. 321, 305

Haardt, F. and Maraschi, L.: 1993, Astrophys.J. 413, 507

Haardt, F. and Matt, G.: 1993, Monthly Not. Roy. Astr. Soc. 261, 346

Nagirner, D.I. and Poutanen, J.: 1993a, Astron.Astrophys. 275, 325

Nagirner, D.I. and Poutanen, J.: 1993b, in: R.A. Sunyaev (ed.) Sov. Sci. Rev. E Astrophys. and Space Phys. 9, 1

Poutanen, J. and Vilhu, O.: 1993, Astron.Astrophys. 275, 337

Sunyaev, R.A. and Titarchuk, L.G.: 1985, Astron.Astrophys. 143, 374

Wandel, A. and Liang, E.P.: 1991, Astrophys.J. 380, 84

472

T. J.-L. Courvoisier and A. Blecha: Multi-Wavelength Continuum Emission of AGN, 472.

(C) 1994 IAU. Printed in the Netherlands. 